\title{
Secure Multiuser MISO Communication Systems with Quantized Feedback
}

\author{
Berna Özbek and Özgecan Özdoğan \\ Department of Electrical and Electronics Engineering \\ Izmir Institute of Technology \\ Izmir, Turkey \\ Email:\{bernaozbek,ozgecanozdogan\}@iyte.edu.tr
}

\author{
Güneş Karabulut Kurt \\ Wireless Communications Reserach Laboratory \\ Department of Electronics and Communication Engineering \\ Istanbul Technical University, Istanbul, Turkey \\ Email: gkurt@itu.edu.tr
}

\begin{abstract}
Physical layer security is a promising approach to provide secure communications by considering the characteristics of wireless channels. In this work, we propose a secure multiple input single output (MISO) multiuser system with a quantized feedback link. We assume that eavesdropper is passive and its channel state information (CSI) is not available at transmitter. In order to disrupt reception of a passive eavesdropper, we schedule more than one legitimate user. For the sake of ensuring secure communication, the CSI of legitimate users has great impact on overall performance of secrecy sum capacity. The proposed solution applies a semi-orthogonal selection with a specific codebook to reduce the quantization errors for legitimate user side while disrupting the reception of the eavesdropper. The proposed solution improved secrecy sum capacity while reducing the feedback overhead for secure MISO multiser system.
\end{abstract}

\section{INTRODUCTION}

Information security is a critical issue in wireless communication due to the inherent open nature of wireless medium and it has received considerable attention. The problem of secure communication so called wiretap channel first has been introduced by Wyner [1]. In this pioneering work, a point to point communication has been considered, i.e., there are three terminals, one sender, one receiver and one eavesdropper. Physical layer security techniques that exploit different characteristics of wireless communication channels (e.g., fading, noise, interference and multiple antenna techniques that enable secure multiuser communication) improve the overall performance for secure systems.

Secrecy capacity can be enhanced by using multiple antenna techniques. For secure single-user multiple input single output (MISO) systems, an artificial noise (AN) has been added to increase secrecy capacity in [2]. In this approach, the transmitter injects AN into the null space of channel of legitimate user without affecting intended user. Thus, it disrupts the reception of eavesdropper that maliciously try to attain information from legitimate user's signal. However, the transmit power is partitioned between the information signal and AN to mask the desired signal from any potential eavesdropper. When the transmit power is allocated properly, a positive secrecy capacity can be guaranteed even if eavesdropper has better channel conditions than the legitimate user.

The secrecy capacity can be improved by serving multiple legitimate users simultaneously in multiuser MISO systems.
In order to schedule multiple legitimate users, zero forcing beamforming (ZFBF) is performed to mitigate the effects of inter-user interference. Subsequently, the multiuser systems with security considerations have been examined in [3]- [6].

The existence of eavesdropper's channel state information (CSI) at transmitter has a great impact on the system secrecy capacity in both single-user and multiuser communications. In the single-user case, the CSI of eavesdropper is assumed to be known perfectly known at transmitter in [7]- [9]. In the case of unknown CSI of eavesdropper, a secure single-user MISO systems by adding AN with limited feedback has been analysed in [10].

Ergodic secrecy sum capacity for multiuser MISO have been investigated and closed form expressions have been obtained depending on the CSI of eavesdropper at the transmitter. The presence of external passive eavesdropper that we have only statistical knowledge of it, has been discussed in [11]. Likewise, the case of internal eavesdropper with imperfect CSI at the transmitter has been covered in [12] [13]. While protecting confidential messages, no information regarding the eavesdropper has been presumed at the transmitter, which is highly probable scenario in practical cases, since the network can be attacked by eavesdroppers that listen legitimate users passively without revealing their CSI.

For multiuser MISO systems, the CSI of the legitimate users are required at the transmitter to perform ZFBF. It is not possible to completely eliminate the effect of interuser interference in the case of quantized feedback and the inter-user interference has a pivotal role in multiuser MISO communication. However, it can be used as an useful tool to disrupt reception of any possible eavesdropper for secure multiuser MISO systems. A large number of scheduling legitimate users will result higher inter-user interference, resulting in a reduction in the capacity of the legitimate users. On the other hand, the interference between the legitimate users will disrupt eavesdropper transmission and thus it degrades the performance of eavesdropper as in the case of using AN without allocation any power [14].

In this work, we consider a cellular layout including a secure multiuser MISO system. We propose to use a semiorthogonal selection with a specific codebook at the legitimate users' side to reduce quantization error. Thus, we schedule 


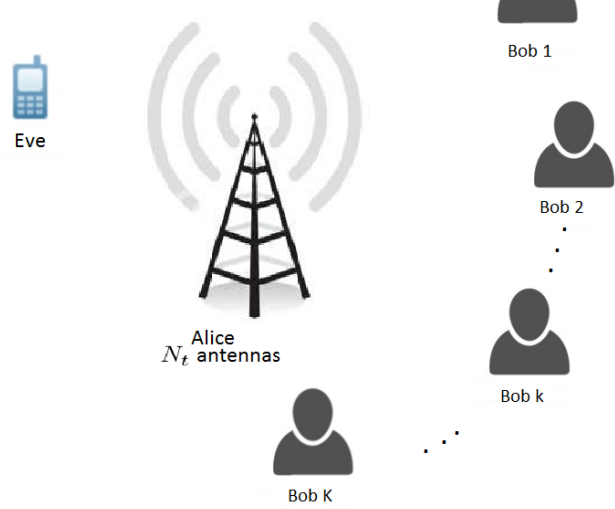

Fig. 1: Secure multiuser MISO downlink communication system

legitimate users having good channel conditions to increase secrecy sum capacity and provide AN effect for eavesdropper while reducing the feedback load significantly.

The rest of this paper is organized as follows. Section II describes the system model for secure multiuser MISO systems. Section III gives a semi orthogonal selection by employing a specific codebook. Section IV explains secure MISO system that includes only one legitimate user. Section V examines performance results and discussions. Finally, section VI gives the conclusions.

\section{SySTEM MODEL}

We consider a multiuser MISO downlink system operating under secrecy constraints as illustrated in Figure 1. The transmitter (Alice) is equipped with $N_{t}$ transmit antennas. The aim of the transmitter is to send confidential messages to intended $M \leq N_{t}$ legitimate users (Bobs) for secure communication from the set of active $K>M$ users. We assume that there is an eavesdropper (Eve) whose aim is to attain information from these scheduled $M$ users. Also, the eavesdropper is passive and its CSI is not available at the transmitter. All these $K$ legitimate users and the eavesdropper have only one receive antenna.

The transmitted signal $\mathbf{x}$ at Alice is expressed as,

$$
\mathbf{x}=\hat{\mathbf{W}} \mathbf{s},
$$

where $\mathbf{s}=\left[s_{1}, s_{2}, \ldots, s_{M}\right]$ is an information symbol vector with $\mathbb{E}\left\{\|\mathbf{s}\|^{2}\right\} \leq P$ and $\hat{\mathbf{W}}=\left[\hat{\mathbf{w}}_{1}, \hat{\mathbf{w}}_{2} \ldots, \hat{\mathbf{w}}_{m}, \ldots, \hat{\mathbf{w}}_{M}\right]$ is the precoding matrix generated by employing ZFBF as $\hat{\mathbf{W}}=$ $\hat{\mathbf{H}}(\mathbb{M})^{H}\left(\hat{\mathbf{H}}(\mathbb{M}) \hat{\mathbf{H}}(\mathbb{M})^{H}\right)^{-1}$. The channel matrix, $\hat{\mathbf{H}}(\mathbb{M}) \in$ $\mathcal{C}^{M \times N_{t}}$, includes channel vectors of scheduled legitimate users as $\hat{\mathbf{H}}(\mathbb{M})=\left[\hat{\mathbf{h}}_{1}, \hat{\mathbf{h}}_{2} \ldots, \hat{\mathbf{h}}_{m}, \ldots, \hat{\mathbf{h}}_{M}\right]^{T}$ with $\hat{\mathbf{h}}_{m}$ is the quantized version of channel vector of $m^{\text {th }}$ legitimate user. This channel vector, $\mathbf{h}_{m} \in \mathcal{C}^{N_{t} \times 1}$, is modelled by $\mathcal{C N}(0, \mathbf{I})$.
The columns of $\hat{\mathbf{W}}$ is normalized as $\left\|\hat{\mathbf{w}}_{m}\right\|=1$. Thus, we send confidential messages to $M$ scheduled legitimate users simultaneously while mitigating inter-user interference by ZFBF.

In order to address the lack of perfect CSI for Bobs at the transmitter, it is possible to quantize channel direction information (CDI), $\overline{\mathbf{h}}_{k}=\frac{\mathbf{h}_{k}}{\left\|\mathbf{h}_{k}\right\|}$, and channel quality information (CQI), || $\mathbf{h}_{k} \|$ where $k=1, \ldots, K$. In this work, we assume that CQI is perfectly known at the transmitter. Therefore, each legitimate user quantizes its CDI to an unit norm vector selected from a predetermined codebook with the size of $2^{B}$ where $B$ is the number of quantization bits.

The codebook defined by $\mathbf{C}_{k}=\left\{\hat{\overline{\mathbf{h}}}_{k_{1}}, \hat{\overline{\mathbf{h}}}_{k_{2}}, \ldots, \hat{\overline{\mathbf{h}}}_{2^{B}}\right\}$ can be known at each user separately. Then, each user chooses an optimal codeword to quantize its CDI according to following criterion:

$$
k_{j^{*}}=\arg \max _{1 \leq j \leq 2^{B}}\left|\overline{\mathbf{h}}_{k}^{H} \hat{\overline{\mathbf{h}}}_{k_{j}}\right|
$$

We construct the quantized channel vector for each legitimate user by $\hat{\mathbf{h}}_{k}=\overline{\overline{\mathbf{h}}}_{k_{j^{*}}}|| \mathbf{h}_{k} \|$. The $M$ best legitimate users based on their channel norm are scheduled at Alice.

Then, the received signal at the $m$ th Bob is written as,

$$
y_{b_{m}}=\left\|\mathbf{h}_{b_{m}}\right\|\left(\overline{\mathbf{h}}_{b_{m}}^{H} \hat{\mathbf{w}}_{m}\right) s_{m}+\sum_{j=1, j \neq m}^{M}\left\|\mathbf{h}_{b_{m}}\right\|\left(\overline{\mathbf{h}}_{b_{m}}^{H} \hat{\mathbf{w}}_{j}\right) s_{j}+n_{b_{m}},
$$

where $n_{b_{m}}$ is additive white Gaussian noise (AWGN) with zero mean and variance of $\sigma^{2}$.

The received signal belonging to the $m$ th Bob at Eve is expressed by

$$
y_{e_{m}}=\mathbf{h}_{e}^{H} \hat{\mathbf{w}}_{m} s_{m}+\sum_{j=1, j \neq m}^{M} \mathbf{h}_{e}^{H} \hat{\mathbf{w}}_{j} s_{j}+n_{e} .
$$

where $n_{e}$ is AWGN with zero mean and variance of $\sigma_{e}^{2}$.

The signal-to-interference-noise ratio (SINR) at $m$ th Bob is written as,

$$
\hat{\gamma}_{b_{m}}=\frac{\frac{P}{M}|| \mathbf{h}_{b_{m}}||^{2}\left|\overline{\mathbf{h}}_{b_{m}}^{H} \hat{\mathbf{w}}_{m}\right|^{2}}{\sum_{j=1, j \neq m}^{M} \frac{P}{M}|| \mathbf{h}_{b_{m}}||^{2}\left|\overline{\mathbf{h}}_{b_{m}}^{H} \hat{\mathbf{w}}_{j}\right|^{2}+\sigma^{2}},
$$

The SINR at Eve belonging to the $m$ th Bob is given by,

$$
\hat{\gamma}_{e_{m}}=\frac{\frac{P}{M}\left|\mathbf{h}_{e}^{H} \hat{\mathbf{w}}_{m}\right|^{2}}{\sum_{j=1, j \neq m}^{M} \frac{P}{M}\left|\mathbf{h}_{e}^{H} \hat{\mathbf{w}}_{j}\right|^{2}+\sigma_{e}^{2}} .
$$

Then, the secrecy sum capacity under quantized CDI is determined in the following:

$$
R=\sum_{m=1}^{M} \mathbb{E}\left\{\log _{2}\left(1+\hat{\gamma}_{b_{m}}\right)\right\}-\mathbb{E}\left\{\log _{2}\left(1+\hat{\gamma}_{e_{m}}\right)\right\} .
$$

Since $\hat{\mathbf{W}}$ is not perfectly orthogonal to the channel of legitimate users, inter-user interference negatively affects the reception of Bobs. In the meantime, inter-user interference degrades the reception of the Eve. In order to reduce the quantization error for legitimate users, we propose to apply the 
semi-orthogonal selection criterion with specific codebook at Bobs' side. Due to the fact that inter-user interference can not be completely eliminated, the reception of Eve is still disturbed in the proposed solution.

\section{THE SEMI-ORTHOGONAL SELECTION WITH SPECIFIC CODEBOOK}

In order to maximize the secrecy sum capacity of the downlink MISO system while achieving multiuser diversity, it is necessary to select the best combinations of $M$ legitimate users. Therefore, the legitimate users having poor channel condition (have low norm and/or causes high inter-user interference) should not take part in the scheduling algorithm, nor feedback their CSI.

Inter-user interference is a pivotal factor in classical multiuser communications. On the other hand, it may have a positive impact for secure multiuser systems since it causes interference to eavesdropper transmission. Therefore, the selection criterion at the Bobs' side is very critical to improve secrecy sum capacity. In this work, we propose to apply semiorthogonal criterion with a specific codebook at the legitimate user side. Then, only those users are fed back their CSI to Alice by using a specific codebook. Along with that, we schedule best $M$ legitimate users according to their norms to achieve higher secrecy sum capacity.

The semi-orthogonal criterion selects the legitimate users whose CDI are semi-orthogonal. Each Bob generates the same $N_{t}$ random orthonormal vectors as $\phi_{i} \in \mathcal{C}^{N_{t} \times 1}, i=1, \ldots, N_{t}$. Then, they measure the orthogonality between their channels and $\phi_{i}$ using the chordal distance:

$$
d^{2}\left(\overline{\mathbf{h}}_{k}, \boldsymbol{\phi}_{i}\right)=1-\left|\overline{\mathbf{h}}_{k}^{H} \boldsymbol{\phi}_{i}\right|^{2}
$$

Let $\mathcal{O}^{N_{t}}$ be the unit sphere lying in $\mathcal{C}^{N_{t}}$ and centered at the origin. Using the chordal distance metric, for any $0<\epsilon<1$, we can define a spherical cap on $\mathcal{O}^{N_{t}}$ with center o and square radius $\epsilon$ as the open set :

$$
\mathcal{B}_{\epsilon}(\mathbf{o})=\left\{\overline{\mathbf{h}}_{k} \in \mathcal{O}^{N_{t}}: d^{2}\left(\overline{\mathbf{h}}_{k}, \mathbf{o}\right) \leq \epsilon\right\}
$$
[15]:

Then, we apply the criterion $\mathcal{T}_{3}$ as described in the following

$$
\mathcal{T}_{3}=\left\{k \in K: \overline{\mathbf{h}}_{k} \in \bigcup_{i=1}^{N_{t}} \mathcal{B}_{\epsilon}\left(\phi_{i}\right) \text { and }\left\|\mathbf{h}_{k}\right\|^{2} \geq \gamma_{t h}\right\}
$$

where $\epsilon$ is the threshold on semi-orthogonality criterion and $\gamma_{t h}$ is the threshold on norm. Thus, the legitimate users which satisfy semi-orthogonality condition but having low norm should not take part in scheduling for reason that channel quality of selected Bobs directly affects the secrecy sum capacity.

Consequently, $\bar{K}$ Bobs on average are allowed to fed back their CSI to Alice whose schedules $M$ legitimate users with the highest norm to establish secure transmission.

The threshold values are determined to have $\bar{K}$ legitimate users in average in limited feedback link by,

$$
\bar{K}=K N_{t} \sum_{b=0}^{N_{t}-1} \frac{\exp \left(-\gamma_{t h}\right)\left(\gamma_{t h}\right)^{b}}{b !} \epsilon^{N_{t}-1}
$$

In order to reduce the quantization error for the legitimate users selected by the criterion $\mathcal{T}_{3}$, we use a specific codebook based on the quantization of the localized region [15] instead of the normalized independent and identically distributed (i.i.d) channel in isotropically distributed in $\mathcal{O}^{N_{t}}$ [16]. As in the i.i.d. case, we use a practical vector quantization scheme to design the local packings. For the $\mathcal{T}_{3}$ criterion, the codebook is adapted according to the orthogonal vectors $\phi_{i}$. From the local packing associated to the spherical cap $\mathcal{B}_{\epsilon}(\mathbf{o})$, it is possible to compute $\mathcal{B}_{\epsilon}\left(\phi_{i}\right)$ using the rotation matrix:

$$
\phi_{i}=\mathbf{U}_{r} \mathbf{o}
$$

where $\mathbf{U}_{r}$ is the unitary rotation matrix.

\section{SCHEDULING ONLY ONE LEGITIMATE USER}

In order to illustrate that the proposed solution achieves a performance gain compared to the case where only the best legitimate user is scheduled rather than $M$ best legitimate users, we define the quantized secrecy capacity for the singleuser secure MISO systems including AN to disturb Eve's reception.

In the case of quantized CDI, transmitted signal masked with AN for single user can be expressed as,

$$
\mathbf{x}_{k}=\hat{\mathbf{f}}_{k} s_{k}+\hat{\mathbf{Q}}_{k} \mathbf{a},
$$

where $s_{k}$ is the information-bearing signal for $k$ th $\mathrm{Bob}$, $\hat{\mathbf{f}}_{k} \in \mathcal{C}^{N_{t} \times 1}$ is the precoding vector, $\mathbf{a}=\left[a_{1}, a_{2}, \ldots, a_{N_{t}-1}\right]^{T}$ represents the $\mathrm{AN}$ vector whose entries are i.i.d. complex Gaussian distributed, $\hat{\mathbf{Q}}_{k} \in \mathcal{C}^{N_{t} \times N_{t}-1}$ is the AN beamformer with orthonormal columns. The beamformers $\hat{\mathbf{f}}_{k}$ and $\hat{\mathbf{Q}}_{k}$ are determined through quantized CSI, respectively, as $\hat{\mathbf{f}}_{k}=\hat{\overline{\mathbf{h}}}_{k}$ and $\hat{\mathbf{f}}_{k} \hat{\mathbf{Q}}_{k}=\mathbf{0}_{1 \times N_{t}-1}$.

Thus, the received signal at $k$ th Bob and Eve can be written respectively,

$$
\begin{gathered}
y_{k}=\left\|\mathbf{h}_{k}\right\|\left(\left(\overline{\mathbf{h}}_{k}\right)^{H} \hat{\overline{\mathbf{h}}}_{k}\right) s_{k}+\left\|\mathbf{h}_{k}\right\|\left(\left(\overline{\mathbf{h}}_{k}\right)^{H} \hat{\mathbf{Q}}_{k}\right) \mathbf{a}+n_{k}, \\
y_{e}=\mathbf{h}_{e}^{H} \hat{\overline{\mathbf{h}}}_{k} s_{k}+\mathbf{h}_{e}^{H} \hat{\mathbf{Q}}_{k} \mathbf{a}+n_{e} .
\end{gathered}
$$

where $n_{k}$ is additive white Gaussian noise with zero mean and variance of $\sigma^{2}$.

Thus, the SINR at $k$ th Bob is given by,

$$
\hat{\gamma}_{k}=\frac{\left\|\mathbf{h}_{k}\right\|^{2}\left|\left(\overline{\mathbf{h}}_{k}\right)^{H} \hat{\overline{\mathbf{h}}}_{k}\right|^{2} a P}{\left\|\mathbf{h}_{k}\right\|^{2}\left|\left(\overline{\mathbf{h}}_{k}\right)^{H} \hat{\mathbf{Q}}_{k}\right|^{2} \frac{1-a}{N_{t}-1} P+\sigma^{2}},
$$

The SINR at Eve can be expressed as,

$$
\hat{\gamma}_{e}=\frac{\left|\left(\mathbf{h}_{e}\right)^{H} \hat{\overline{\mathbf{h}}}_{k}\right|^{2} a P}{\left|\left(\mathbf{h}_{e}\right)^{H} \hat{\mathbf{Q}}_{k}\right|^{2} \frac{1-a}{N_{t}-1} P+\sigma_{e}^{2}},
$$

where $P$ is the total power, $a$ denotes the power allocation parameter between transmitted message and AN signal. 
Then, secrecy capacity in quantized case for single user is given as

$$
R_{b}=\mathbb{E}\left\{\log _{2}\left(1+\hat{\gamma}_{k}\right)\right\}-\mathbb{E}\left\{\log _{2}\left(1+\hat{\gamma}_{e}\right)\right\} .
$$

In contrast to the case that CSI is perfectly known at transmitter, an AN leakage occurs in the quantized feedback case. This AN leakage term, || $\mathbf{h}_{k}||^{2}\left|\left(\overline{\mathbf{h}}_{k}\right)^{H} \hat{\mathbf{Q}}_{k}\right|^{2}$ in Eq. (16), negatively affects the reception of legitimate user and it reduces the secrecy capacity in Eq. (18).

\section{Simulation Results}

We illustrate the simulation results for $N_{t}=2$ transmit antennas at Alice. The pair of $\left(\gamma_{t h}, \epsilon\right)$ for $\mathcal{T}_{3}$ criterion is calculated to have an average number of users in the cell $\bar{K}=4$. Then, the pairs are chosen as $[(1.65,0.4),(2,0.25),(2.3,0.2),(2.55,0.18),(2.6,0.15)]$.

Only the legitimate users that satisfy these thresholds are fed back their $B$ bits corresponding to the codebook index of their quantized CDI to Alice. Exploiting the feedback information, Alice schedules the $M$ legitimate users by performing ZFBF to reduce inter-user interference. In this work, the worst case scenario which means that no knowledge about CSI of Eve is available at Alice is considered.

The secrecy sum capacity comparison for $\mathcal{T}_{3}$ criterion with semi orthogonal selection and full feedback case in which all users are fed back their CSI without any selection at the legitimate user side are illustrated in Figure 2 and Figure 3 depending on the number of active users and signal-to-noise ratio (SNR). In Figure 4, the feedback load is compared for full feedback and $\mathcal{T}_{3}$ criterion. The overhead is reduced from $60 \%$ to $92 \%$ through $\mathcal{T}_{3}$ criterion based on the number of the active legitimate users in the system in the expense of reduction on secrecy sum capacity between $0.1 \mathrm{bps} / \mathrm{Hz}$ and $1 \mathrm{bps} / \mathrm{Hz}$. It is observed that when the number of quantization bits are increased, the gap on secrecy sum capacity for full feedback and $\mathcal{T}_{3}$ criterion is also increased.

In Figure 5 and Figure 6, we compare the secrecy sum capacity for $\mathcal{T}_{3}$ criterion with special codebook. The performance results indicate that the secrecy sum capacity improves between $2.1 \mathrm{bps} / \mathrm{Hz}$ and $0.4 \mathrm{bps} / \mathrm{Hz}$ depending on the number of quantization bits for $K=50$ by employing the special codebook. When the number of quantization bits is increased, the gain on secrecy sum capacity becomes low since the disturbance effect of inter-user interference on Eve is also reduced. Since the secrecy sum capacity is improved significantly especially at low feedback load, the semi-orthogonal criterion with a special codebook leads to a promising solution for practical applications.

For secure single user MISO systems with perfect CSI case, the power is shared equally between message signal and AN as an optimal manner. However, for the quantized case, as shown in Figure 7, when the number of quantization bits are reduced, the power allocation parameter $\alpha$ should be increased to maximize secrecy capacity. As a result, as the number of quantization bits reduces, most of the available power

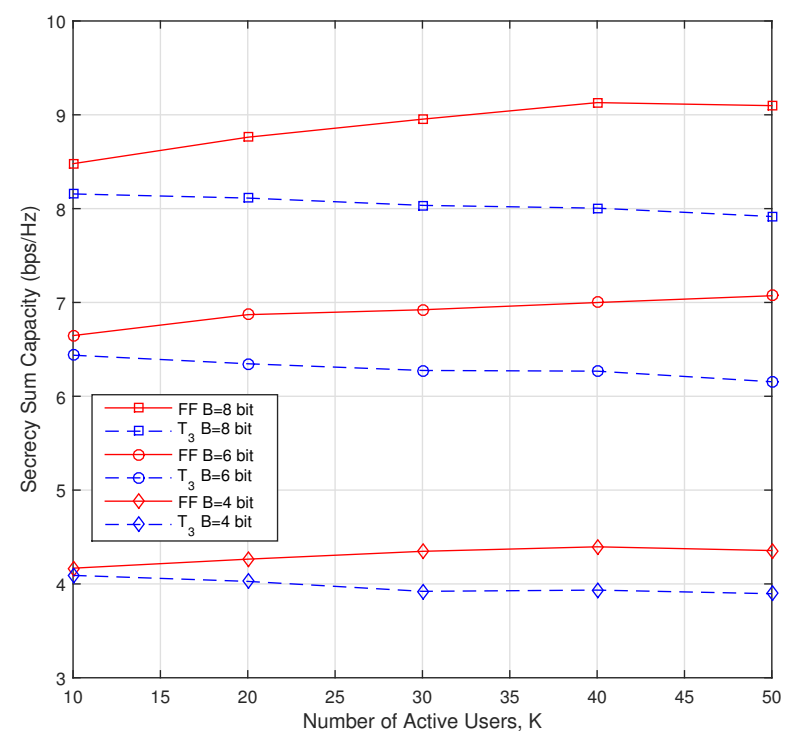

Fig. 2: The comparison between full feedback $(\mathrm{FF})$ and $\mathcal{T}_{3}$ criterion at $\mathrm{SNR}=20 \mathrm{~dB}$ for the different number of active users.

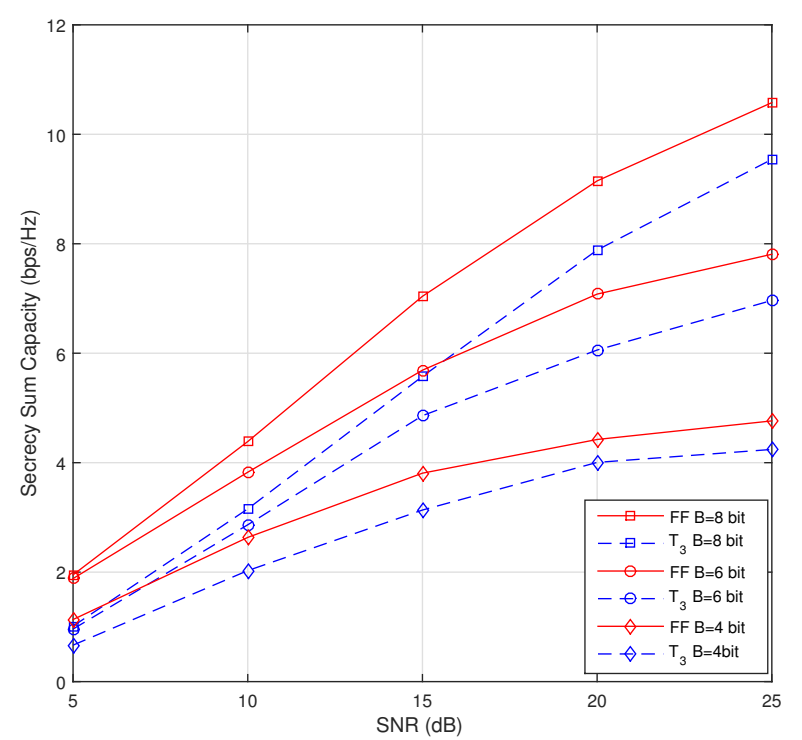

Fig. 3: The comparison between full feedback $(\mathrm{FF})$ and $\mathcal{T}_{3}$ criterion at $K=50$ for different SNR values.

at transmitter should be used for transmitting information message rather than AN.

In Figure 8, we compare the secrecy capacity for the system that performs semi-orthogonal selection having specific codebook and the system that schedules only one legitimate user with AN having the optimal power allocation based on Figure 7. The performance results are shown that the proposed solution for multiuser MISO system provides much better 


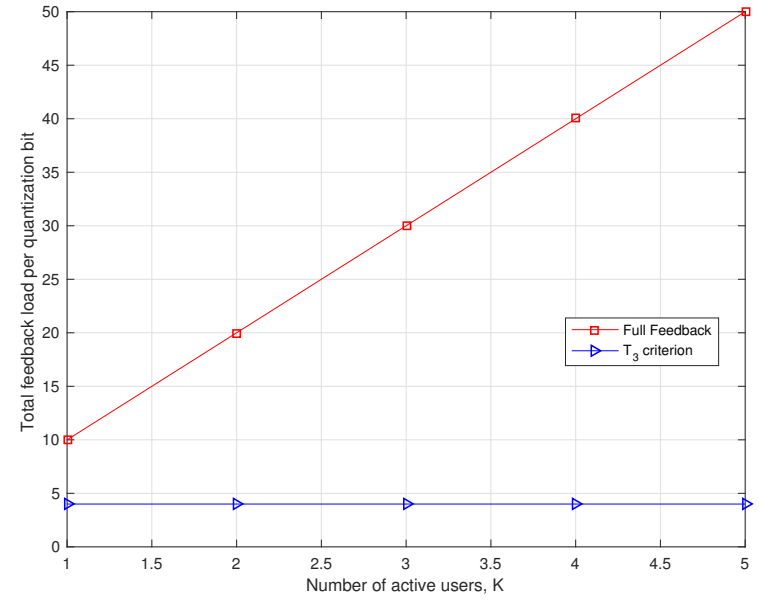

Fig. 4: The total feedback load per the number of feedback bit for full feedback (FF) and $\mathcal{T}_{3}$ criterion.

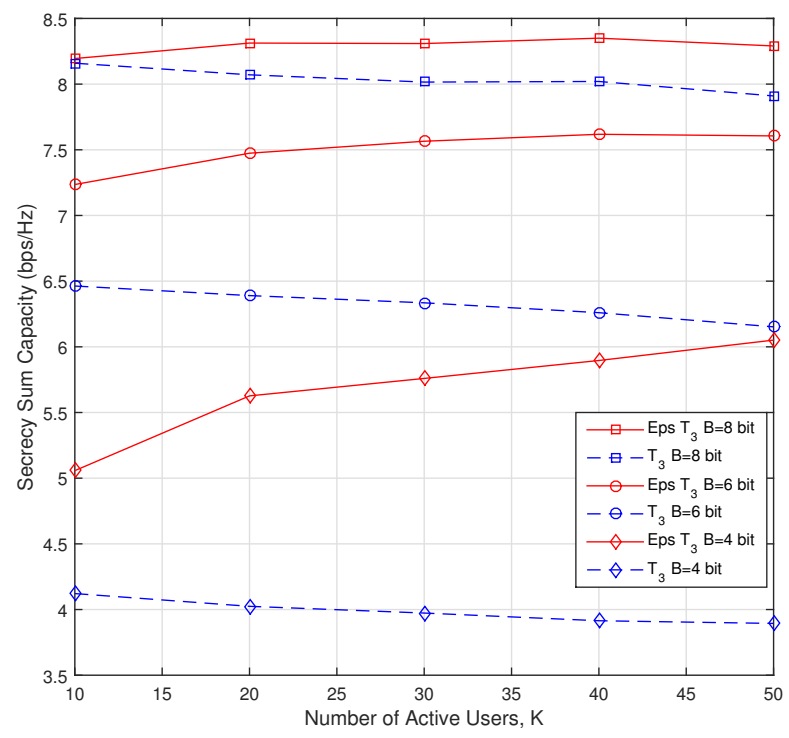

Fig. 5: The comparison between $\mathcal{T}_{3}$ criterion with and without a special codebook design for $\mathrm{SNR}=20 \mathrm{~dB}$.

secrecy capacity performance than single user MISO system while requiring much less overhead through feedback link.

\section{CONCLUSION}

In this work, we have applied the semi orthogonal selection with specific codebook at legitimate user side for multiuser MISO systems with secrecy constraints. In practical systems, CSI regarding the eavesdropper is not possible to have at the transmitter side, since eavesdropper is passive, we consider the case that transmitter has no knowledge of eavesdropper CSI. We have scheduled more than one legitimate user at the transmitter side under quantized feedback link to disrupt

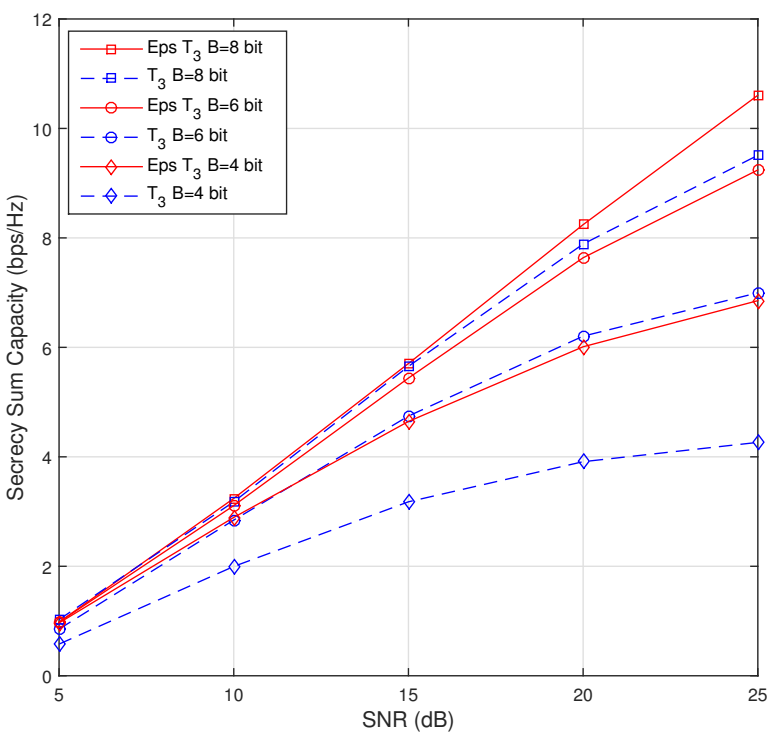

Fig. 6: The comparison between $\mathcal{T}_{3}$ criterion with and without proposed codebook at $K=50$ for different SNR values.

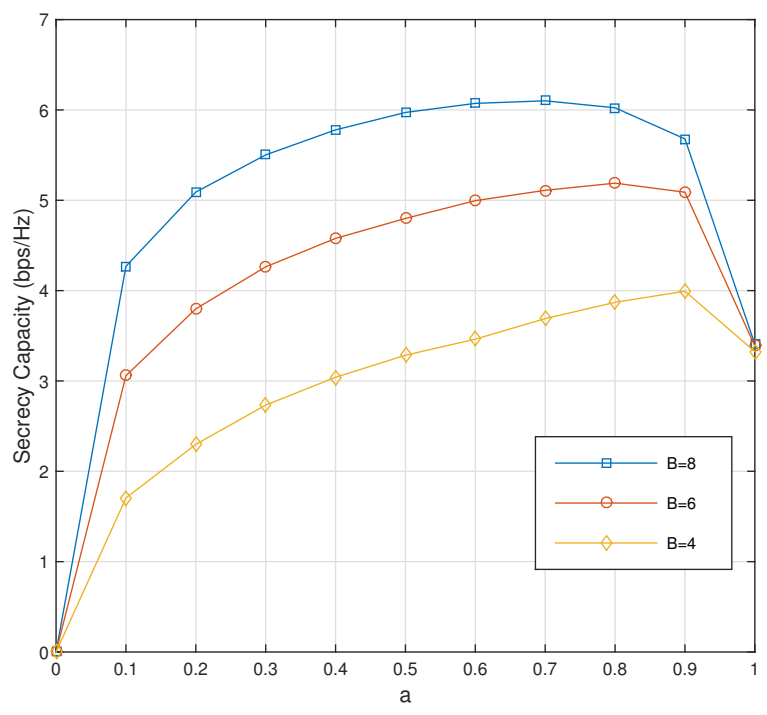

Fig. 7: Power allocation parameter for different number of bits in single user MISO systems at $\mathrm{SNR}=20 \mathrm{~dB}$.

the reception of eavesdropper. By performing semi orthogonal selection, we have reduced the overhead by preventing the legitimate users having poor channel conditions to feed back their CSI. Besides that, we have employed specific codebook thanks to the properties of semi orthogonal selection. We have illustrated that we have increased secrecy sum capacity significantly for low number of quantization bits, which conducts to design physical layer security systems for practical applications. 


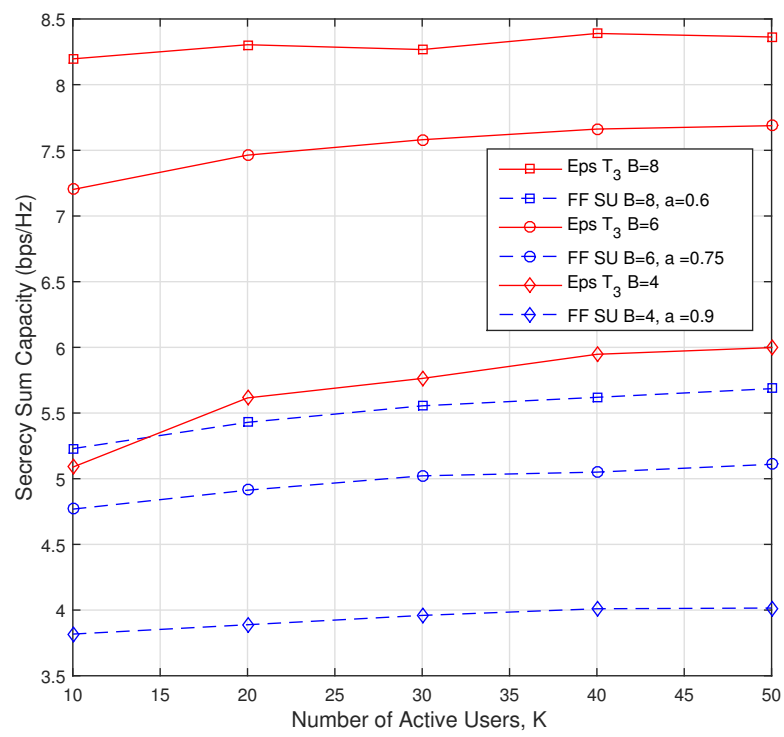

Fig. 8: The comparison between single user MISO with full feedback (FF) case and multiuser MISO with $\mathcal{T}_{3}$ criterion and special codebook at $\mathrm{SNR}=20 \mathrm{~dB}$.

\section{ACKNOWLEDGMENT}

This work has been carried out in the framework of TUBITAK 114E626 Project.

\section{REFERENCES}

[1] A. D. Wyner, The wire-tap channel, The Bell System Technical Journal, vol. 54, no. 8, pp. 1355-1387, Oct. 1975.

[2] S. Goel, R. Negi, Guaranteeing secrecy using artificial noise, IEEE Transactions Wireless Communications, vol. 7, no. 6, pp.2180-2189, June 2008.
[3] I. Csiszar and J. Korner, Broadcast channels with confidential messages, IIEEE Transactions on Information Theory, vol. 24, no. 3, pp. 339-348, May 1978.

[4] Y. Liang and H. V. Poor, Multiple-access channels with confidential messages, IEEE Transactions on Information Theory, vol. 54, no. 3, pp. 976-1002, March 2008.

[5] A. Khisti, A. Tchamkerten, and G. W. Wornell, Secure broadcasting over fading channels, IEEE Transactions on Information Theory, vol. 54, no. 6, pp. 2453-2469, June 2008.

[6] Y. Liang, H. V. Poor, and S. Shamai, Secure communication over fading channels, IEEE Transactions on Information Theory, vol. 54, no. 6, pp. 2470-2492, June 2008

[7] Z. Li,W. Trappe,R. Yates, Secret Communication via Multi-Antenna Transmission, 41st Annual Conference on Information Sciences and Systems, pp. 905-910, Baltimore, 2007.

[8] S. Shafiee and S. Ulukus, Achievable rates in Gaussian MISO channels with secrecy constraints, IEEE ISIT, Nice, France, June 2007.

[9] S. Shafiee, N. Liu, and S. Ulukus, Towards the secrecy capacity of the Gaussian MIMO wire-tap channel: The 2-2-1 channel, IEEE Transactions on Information Theory, vol. 55, no. 9, pp. 4033-4039, Sep. 2009.

[10] X. Zhang, M. R. McKay, X. Zhou, R. W. Heath, Artificial-NoiseAided Secure Multi-Antenna Transmission With Limited Feedback, IEEE Transactions on Wireless Communications, vol:14, issue: 5, pp: 2742 2754, Jan. 2015.

[11] X. Chen,R. Yin, Performance analysis for physical layer security in multi-antenna downlink networks with limited CSI feedback IEEE Wireless Communications Letters, vol. 2 ,no. 5,October 2013.

[12] G. Geraci,R. Couillet, J. Yuan, M. Debbah,I. B. Collings, Secrecy sumrates with regularized channel inversion precoding under imperfect CSI at the transmitter, IEEE ICASSP, Vancouver, 2013.

[13] N. Li, X. Tao, J. Xu, Ergodic secrecy sum-rate for downlink multiuser MIMO systems with limited CSI feedback IEEE Communications Letters, vol.18, No.6, June 2014.

[14] X. Chen, Y. Zhang, "Mode Selection in MU-MIMO downlink networks: a physical layer security perspective" IEEE Systems Journal, doi:10.1109/JSYST.2015.2413843,2015.

[15] B. Ozbek and D. Le Ruyet, Feedback strategies for wireless communication systems, Springer-Engineering Series Book, Springer-Verlag New York, U.S.A, 2014

[16] A. Narula, M. J. Lopez, M. D. Trott, G. W. Wornell, Efficient use of side information in multiple antenna data transmission over fading channels, IEEE Journal on Selected Areas in Communications, vol:16, pp.14231436, October 1998. 